\title{
La evaluación de habilidades de información en la licenciatura de Periodismo en la Universidad Central "Marta Abreu" de Las Villas, Cuba"
}

\author{
Dra. Grizly MENESES-Placeres \\ Universidad Central "Marta Abreu"de Las Villas (Cuba) \\ grizly@uclv.edu.cu
}

Recibido: 03/03/2011

Aceptado: 03/03/2011

\section{RESUMEN}

El trabajo presenta el proceso de evaluación de las habilidades de información de estudiantes de Periodismo. Objetivo: Evaluar la situación existente en los estudiantes de la Licenciatura en Periodismo en cuanto al desarrollo de sus habilidades de información de la Universidad Central "Marta Abreu" de Las Villas (UCLV). Metodología: Se presenta un enfoque mixto donde se conjugan resultados de información de perspectiva cualitativa y cuantitativa. Básicamente se empleó el método de investigación-acción para realizar la evaluación. Dentro de las técnicas se utilizaron el cuestionario y el grupo focal como esenciales. Resultados: La autovaloración que tienen los estudiantes incluidos en la investigación arrojó tener niveles medio y bajos de destreza de habilidades de Información. El escenario docente de la asignatura documentación periodística fue determinante en la transformación de la realidad encontrada en el diagnóstico. Las opiniones dadas por los estudiantes en cada sesión, así como las evaluaciones finales demostraron el avance de los encuestados con respecto al desarrollo de las habilidades de la información.

Palabras clave UCLV; evaluación; habilidades de información; periodismo

The evaluation of information skills in undergraduate journalism Central University "Marta Abreu" de Las Villas, Cuba

\begin{abstract}
The paper presents the information skills assesment of journalism students. Objective: To evaluate the information skills development of Journalism students at the Central University "Marta Abreu" de Las Villas (UCLV). Methods: We present a hybrid approach which combines the qualitative and quantitative perspective. Basically we used the action-research method to conduct the evaluation. Among the techniques used there are the questionnaire and focus group as critical. Results: The self-assessment with students involved in research have determined medium and low levels of information skills. The educational scenario of the subject journalistic documentation, was decisive in the transformation of the reality encountered in the diagnosis. The opinions given by students in each session and final evaluations showed the progress of the respondents with regard to developing the information skills.
\end{abstract}

Key words: UCLV; assessment, information skills, journalism

\footnotetext{
${ }^{1}$ El estudio que se presenta es un resultado parcial de la Tesis Doctoral de la autora, leída en la Universidad de Granada en Diciembre de 2010 y Directora Dra. María Pinto Molina.
} 


\section{INTRODUCCIÓN}

Resulta indudable la influencia de las tecnologías de la información y las comunicaciones en todos los sectores de la sociedad. Incluso es muchos casos se discute si es efectivamente el objeto o centro del proceso más que un medio o herramienta para alcanzar las metas e impulsar el desarrollo.

Las ciencias sociales reconocen al paradigma tecnológico que cada vez con más fuerza se impone. Las materias provenientes del campo de las humanidades, la psicológica, la sociología, las ciencias de la información, la comunicación han estudiado ampliamente el impacto que han tenido las TIC en su radio de acción.

En la presente investigación se analiza otra rama crucial donde las TIC han cambiado los roles, los estilos, los saberes: el periodismo. Aunque se coincide en que este es un campo donde aún se considera a las TIC como un medio o herramienta, los retos están latentes cuando son los periodistas los encargados por excelencia de llevar el mensaje comunicativo verídico a los ciudadanos de la sociedad de la información.

Se desprende entonces, que el nuevo periodista no solo debe dominar las herramientas (TIC), sino que debe profundizar y pulir los mecanismos con los cuales accede a la información que hará verdadero su mensaje. La explosión de los medios digitales -prensa, televisión, radio- hacen necesario la sistematización de temas referidos a cómo localizar y acceder a la información en ambiente web, cómo organizarla de forma coherente, cómo evaluarla, para al final presentar un producto comunicativo eficiente, que promulgue valores éticos en cuanto al uso de la información.

BORSTEIN resume las habilidades de información con las que debe contar un profesional del periodismo. Se basa para ello en las normas y estándares para el acceso y uso de la información para la educación superior. Significativo resulta los escenarios reales en los cuales el periodista tendría que aplicarlas, enfatiza en (BORSTEIN, 2003):

El desarrollo de sus artículos periodísticos

Proveer información de background

Formar contexto a las historias

Identificar fuentes de información expertas o no expertas

Preparación de entrevistas

Localización de estadísticas

Verificación de hechos

Se pretende desarrollar entonces en el futuro profesional habilidades de información que repercutan de forma efectiva en el ejercicio de su profesión. De ahí que se parta se un diagnóstico inicial que proporcione las bases para favorecer dicho desarrollo.Las noticias son el resultado de decisiones realizadas por el periodista sobre la revisión exhaustiva de diversas fuentes de información utilizadas, de un proceso de búsqueda y relevancia de información para ser publicada con posterioridad. Sin embargo, pese a la diversidad de fuentes de información y de metodologías para encontrar información - 
bases de datos, reportes, imágenes- los periodistas a menudo se reducen a la utilización de fuentes personales para constatar hechos y citas (ERICSON et al., 1989)

Así, los periodistas tienen la obligación de asegurar que usaron los mejores y más apropiados métodos de búsqueda de información, verificación y uso para hacer una correcta y actualizada información a la audiencia(BRADLEY, 2003) En la actualidad, con el desarrollo de Internet y todo lo que representó en cuanto a la evolución de las comunicaciones (la aparición de las bases de datos electrónicas, las World Wide Web, la multimedialidad y la instantaneidad de la red, por solo mencionar algunos de sus rasgos más significativos), comenzaron a aparecer nuevas posibilidades para el periodismo. Ahora hay mayor producción de noticias a escala mundial que aparecen casi en su totalidad publicadas en la red. Un problema que aparece por las facilidades del mundo web, los periodistas hoy están confiando más en una fuente electrónica, que en ocasiones puede ser menos fidedigna que cualquier otra fuente, asi tienen la necesidad de desarrollar estándares para verificar con precisión la información digital (CARRISON, 2000). Por tal motivo, poseer habilidades como las anteriormente expuestas y ser verdaderamente competentes es un requisito obligatorio para los profesionales de la prensa. Imprescindible para informar de manera efectiva, es la capacidad de comprender, también presente en los postulados sobre alfabetización informacional. La capacidad de examinar y comprender está “...inherente en el concepto de alfabetización en información..." (BENITO, 2000). La necesidad de entender problemas relacionados con la economía, la política y la realidad social es otro punto dentro de la competencia informacional que mucho tiene que ver con el diarismo.

Según el Modelo Curricular para la enseñanza del Periodismo en los países en desarrollo y las democracias incipientes (UNESCO, 2007), la práctica del reporterismo exige de numerosas competencias. Entre ellas menciona algunas elementales, que van desde la posesión de conocimientos generales y capacidad intelectual hasta dominar las técnicas profesionales de investigación, escritura, edición y diseño. Incluye además la capacidad para utilizar adecuadamente las herramientas del periodismo y adaptarse a las nuevas tecnologías.

El periodismo o el arte de informar, en sí mismo, lleva implícita la competencia en el uso y manejo de la información. La capacidad para comprender, analizar, sintetizar y evaluar los datos de forma rápida y eficaz son características que rodean el ejercicio investigativo $\mathrm{y}$, por tanto, la labor de los periodistas. El diarismo es, básicamente, investigación. El pensamiento crítico, piedra angular de cualquier trabajo informativo, constituye también un elemento invariablemente unido a la competencia informacional. (UNESCO, 2007)

Las habilidades de información intervienen en toda la labor del periodista: los ayuda a identificar y evaluar las fuentes, a localizar estadísticas, verificar hechos, construir historias, redactar noticias. La alfabetización informacional refuerza la limpieza y verosimilitud del reporterismo, debido al conocimiento de los aspectos económicos, sociales y legales que rodean la producción y uso de la información. Además, promueve el respeto a la información y la ética en el uso de la misma, elementos esenciales para realizar un periodismo responsable en el siglo XXI. Esto se reafirma aún cuando la realidad sigue su curso vertiginoso. El estilo continúa siendo la virtualización de los 
medios. Hoy día, las tendencias del periodismo actual van hacia su inserción en las denominadas aplicaciones web 2.0, definida como aquellas que sacan partido a las ventajas intrínsecas de la web, ofreciendo un servicio continuamente actualizado, que mejora cuanto más gente lo use, utilizando y remezclando los datos de múltiples recursos, incluyendo los usuarios individuales, a la vez que ofrecen sus propios datos y servicios de tal forma que pueden ser reutilizados por otros, creando una "arquitectura de participación" en red, yendo más allá de la página de la web 1.0 para ofrecer experiencias de usuario cada vez más ricas. (O' REILLY, 2005)

En el periodismo contemporáneo -véase www.elpais.com, www.elmundo.es o www.abc.es, en aras de promover tal definición se han empezado a conocer algunas características dentro de los medios como reflejos de la web 2.0. Se establece entre otras que (GUALLAR, 2007):

- se busca que los visitantes sean algo más que simples lectores, se invita no solo a opinar sino además a participar en la presentación y la elaboración de los contenidos de los diarios.

- Se incluyen secciones de blogs, canales rss, valoración por los lectores de las informaciones del medio, elaboración de informaciones propias por los lectores.

Un análisis preliminar ubica al usuario y/o lector en el centro de atención del proceso, luego entonces, se impone una interrogante, ¿posee el futuro profesional del periodismo las habilidades de información necesarias para enfrentar los retos que impone las tendencias actuales de la virtualización de los medios?

A partir de esta situación problémica, el presente estudio tiene por objetivo general:

Evaluar la situación existente en los estudiantes de la Licenciatura en Periodismo en cuanto al desarrollo de sus habilidades de información de la UCLV.

La literatura científica dedicada a la evaluación de habilidades de información se considera prolífera. Varios autores han teorizado sobre el particular, que fundamentan los diversos antecedentes que presenta la investigación.

Dentro del proceso de alfabetización informacional la evaluación puede analizarse como eje transversal, pues toca diferentes contenidos en su actividad. Así lo confirman diversos autores (COLBORN y CORDELL, 1998; GRATCH LINDAUER, 2004; IANNUZZI, 1999; PORTMANN y JILIUS ROUSH, 2004)

Otro de los autores, IFLA ${ }^{2}$, establece directrices para la evaluación en la alfabetización informativa, donde se centra exclusivamente en los aprendizajes del estudiante. Para ello, se pronuncia hacia la interrogante ¿qué han aprendido los estudiantes?. Propone entonces tres tipos de evaluación con propósitos diferentes: prescriptiva o diagnóstica, formativa y sumativa.(IFLA, 2004)

\footnotetext{
${ }^{2}$ Federación Internacional de Asociaciones Bibliotecarias
} 
Una revisión de las posturas anteriores, obliga a centrarse en el aprendizaje de los estudiantes como eje medular para evaluar el desarrollo de habilidades informacionales. No obstante la mayoría de los estudios revisados se refieren a la evaluación de programas de Ai. Ejemplos fehacientes de esto, son los estudios realizados por (BURKHARDT, 2007; KUNKEL et al., 1996; LARKIN y PINES, 2005; LAWSON, 1999; MOORE-JANSEN, 1997; MORRISON, 1997; RABINE y CARDWELL, 2000; WONG et al., 2006).

Las revisiones realizadas por (SINGH, 2005) se corresponden con los propósitos del estudio que se presenta; la intención de esta autora fue evaluar las percepciones que tenían las facultades, por ende los programas, de periodismo y comunicación con respecto a la ALFIN a partir de la acreditación de dichos programas por las agencias acreditadoras. Los resultados aseveraron el mejoramiento de los estudiantes en los temas abordados después de las instrucciones recibidas.

\section{METODOLOGÍA}

El estudio siguió un ENFOQUE MIXTO DE INVESTIGACIÓN, justificado en que esta investigación recolecta, analiza y vincula datos cualitativos y cuantitativos para responder al problema de investigación que se pretende resolver (HERNÁNDEZ SAMPIER et al., 2006). Dentro de sus ventajas el propio autor establece:

Se logra una perspectiva más precisa del fenómeno

Potencian la creatividad teórica con suficientes procedimientos críticos

Mejor exploración y explotación de los datos

El contexto de aplicación en puramente universitario, marcado por la selección de la COMUNIDAD DE ESTUDIANTES. Se basó en los siguientes atributos para su determinación:

1. Matriculados en las especialidades objetos de análisis.

2. Mayor cantidad de asignaturas cursadas correspondientes al ejercicio de la profesión.

Comunidad de estudiantes de Periodismo. Fueron escogidos específicamente estos alumnos porque están más próximos a culminar su formación como periodistas y han recibido ya casi la totalidad de las disciplinas presentes en el Plan de Estudios C (perfeccionado). Sus opiniones son especialmente valiosas para la presente investigación: deben tener, precisamente por estar culminando su formación profesional, mayor riqueza y profundidad de criterios con respecto al problema objeto de estudio. Además fueron más accesibles al investigador por fungir como docente en el grupo. Otro elemento que motivó la selección de los informantes fue la composición heterogénea en cuanto al lugar de procedencia de los miembros de ambos grupos: en 
ellos hay estudiantes procedentes de todas las provincias de la región central, desde Camagüey hasta Cienfuegos.

Para la realización exitosa del estudio y tomando en consideración los atributos anteriores, específicamente las comunidades de sujetos son:

Comunidad de estudiantes de las licenciaturas de Periodismo en la Universidad Central "Marta Abreu" de Las Villas

\begin{tabular}{|l|l|l|}
\hline Facultad & Carrera & Grupo \\
\hline Humanidades & Periodismo & 51 \\
\hline
\end{tabular}

Tabla 1. Distribución de encuestados por las especialidades

Comunidad de estudiantes de Periodismo. Se seleccionaron a los estudiantes que ingresaron en la universidad en los cursos 2004-2005 y 2005-2006. En el momento de la investigación cursaban el cuarto y quinto año de la carrera respectivamente. Son 20 alumnos de 4to año y 31 de 5 to para un total de 51 .

Para el desarrollo del trabajo se utilizaron los siguientes MÉTODOS DE INVESTIGACIÓN CIENTÍFICOS. Del nivel teórico:

- Inductivo-Deductivo: sirvió para realizar análisis e inferencias de los datos obtenidos permitiendo arribar a las regularidades y conclusiones presentadas en el estudio.

- Análisis-Síntesis: se utilizó para penetrar en la esencia del fenómeno objeto de estudio y posteriormente realizar una síntesis de la información obtenida.

En el caso del nivel empírico:

- Método de análisis documental. Este método se basa en la recopilación de información a partir de la consulta de fuentes de información diversas. Fundamentalmente es empleado para poder sustentar teóricamente la presente investigación. Las fuentes documentales revisadas, en su mayoría, estuvieron referidas a contrastar los conocimientos en torno a la alfabetización informacional y las prácticas evaluativas en contextos educativos provenientes de Documentos del área de la Educación

- Método de investigación-acción. Dentro de los métodos de la investigación cualitativa, la investigación - acción resulta uno de los más utilizados en las investigaciones relacionadas con las prácticas educativas. Sus inicios se remontan al período de la Segunda Guerra Mundial, cuando Kart Lewin hizo un trabajo donde estableció cuatro fases para su realización: planificar, actuar, observar y reflexionar. (RODRÍGUEZ GOMEZ et al., 2004). En la investigación se aplicó con el propósito de evaluar las habilidades de información en los estudiantes de periodismo.

En el estudio se aplicaron las siguientes TÉCNICAS para la recopilación de datos:

- El grupo focal: Se empleó y organizó con los estudiantes Tuvo las siguientes particularidades: 


\section{Objetivos:}

- Reconocer el dominio de las habilidades de información que poseen los participantes

- Identificar las disciplinas y asignaturas del plan de estudios de la carrera que tributan a la formación de habilidades de información

- Indagar acerca de la visión que tienen sobre el claustro con respecto a sus habilidades de información

- Identificar el rol de la institución de información con respecto al desarrollo y formación de habilidades de información.

\section{Procedimiento:}

Se organizaron en dos sesiones de trabajo. Los aspectos a debatir fueron:

Sesión 1:

- Saber cuáles son las habilidades de información que conocen y su estado de seguridad o ignorancia en cada una. Con el propósito de identificar las habilidades más fuertes y débiles que presenta el alumnado.

Los datos obtenidos en esta primera parte se triangularon con la aplicación del cuestionario ALFINHUMA. (PINTO MOLINA, 2010)

\section{Sesión 2:}

- Qué disciplinas, asignaturas o contenidos específicos han favorecido el desarrollo de habilidades de información. Cuáles consideran que no lo han hecho y pudieran haberlo realizado.

La planificación y realización de los grupos focales se resumen en la siguiente tabla:

\begin{tabular}{|l|l|l|ll|ll|}
\hline $\begin{array}{l}\text { GRUPO } \\
\text { FOCAL }\end{array}$ & SESION & ESPECIALIDAD & FECHA & \multicolumn{2}{|l|}{$\begin{array}{l}\text { DURACION } \\
\text { TEMPORAL }\end{array}$} \\
\hline 1 & $1 \mathrm{ra}$ & Periodismo & $\begin{array}{l}11 \\
2009\end{array}$ & mayo & $\begin{array}{l}8: 40 \text { AM } \\
10: 00 \mathrm{AM}\end{array}$ & hasta \\
\hline 2 & $2 \mathrm{da}$ & Periodismo & 18 & mayo & $\begin{array}{l}10: 05 \text { am } \\
11: 40 \mathrm{am}\end{array}$ & hasta \\
\hline
\end{tabular}

Tabla 2. Cronograma de las sesiones del grupo focal

- El cuestionario: se utilizó con el propósito de identificar el dominio de las habilidades de información presentes en los estudiantes. Se aplicó el cuestionario ALFINHUMA $^{3}$ diseñado para estudiantes universitarios de Ciencias Sociales. El instrumento tiene el objetivo proveer de un mejor conocimiento sobre los niveles de ALFIN a través del significado de variables, categorías y dimensiones para su compresión (PINTO MOLINA, 2010) consistía en una valoración sobre tres variables: importancia, nivel y adquisición, que se refieren a los si-

3 Pertenece al proyecto de investigación de I+D La alfabetización en información para el aprendizaje virtual en el área de humanidades en el marco del Espacio Europeo de Enseñanza Superior. Disponible en www.mariapinto.es/mainframe.html 
guientes aspectos: Importancia (Valorar la importancia que tienen las habilidades de información para su desarrollo académico); Nivel (Valorar el grado de destreza en las referidas habilidades); Adquisición (Dónde ha adquirido esas habilidades). En una escala de menor a mayor que va del 1 (baja) al 9 (excelente), el estudiante ponderaría su valor.

Las habilidades de información fueron agrupadas en cuatro variables en correspondencia con los estándares internacionales revisados - ALA, CAUL, ACRL-, dando como resultado un total de 27 interrogantes:

- Búsqueda de información

- Evaluación de información

- Tratamiento de información

- Comunicación y difusión de información

- La observación fue utilizada en cada intercambio realizado con los informantes escogidos para el estudio, en tanto permite obtener información sobre un fenómeno o acontecimiento tal y como este se produce (RODRÍGUEZ GOMEZ et al., 2004). Sin embargo, se definió como objeto de observación: nivel de destreza de las habilidades de información. Así se determinó que el escenario de la docencia directa impartido por investigadora en cada grupo fuese el escenario propicio para su realización. Las formas de docencia empleadas durante el período donde se efectuó la observación contribuyeron de manera significativa en la pesquisa

Las fases de la investigación, estuvieron determinadas en gran medida por el predominio del método de investigación acción aplicado. Estas etapas fueron:

1. Autoevaluación de las Habilidades de Información en los estudiantes de periodismo. La intención fue recopilar información referida al dominio de las habilidades informacionales presentes en las comunidades de estudiantes escogidas en correspondencia con los niveles de autoevaloración expresado por los estudiantes. Todo el procesamiento se hizo por mediación de métodos matemáticos, con la utilización del Excel y del software estadístico SPSS v.17.0, donde se manejaron estadísticos descriptivos como la media, mediana, moda y medidas de dispersión (desviación típica). Se calcularon las tablas de frecuencia para las diversas variables, y se hicieron correlaciones bivariadas aplicando específicamente el coeficiente de Pearson.

2. Sesiones de las asignaturas. Como parte del currículo de la especialidad se escogió la asignatura donde la investigadora desempeñaba su rol de docente. El eje central era el desarrollo de habilidades de información. Se contó entonces con el espacio de la asignatura Documentación Periodística para actuar. Tenía un total de $42 \mathrm{~h} / \mathrm{c}$ lo que significaba un encuentro directo con los informantes como mínimo con una frecuencia semanal durante el semestre. Por tanto, las acciones directas llevadas a cabo para formar y medir las habilidades información fueron 22 sesiones en total.

3. Resultados de las acciones con los estudiantes. La tercera -y última- fase del estudio tuvo como propósito exponer las evidencias de la transformación o indicios de cambios en los objetos de evaluación. 


\section{ANÁLISIS Y DISCUSIÓN}

\section{Resultados de la Fase 1: Autoevaluación de las Habilidades de Información en los estudiantes de periodismo}

La encuesta aplicada a los alumnos de cuarto y quinto año de la carrera de Periodismo arrojó resultados esclarecedores. A través de ella se midieron aspectos relacionados con la importancia que le confieren los futuros periodistas a la búsqueda, evaluación, tratamiento y presentación de la información. También se les inquirió acerca del nivel de destreza que consideran poseen en las mencionadas habilidades y dónde las han adquirido. Incluido en la búsqueda de información se analizaron cuestiones concernientes a la identificación de las necesidades de información y de las fuentes, además de aspectos relacionados con la localización y acceso a las mismas.

El 85\% de los estudiantes le otorgan una alta importancia a la búsqueda de información. La gran mayoría reconoció la necesidad de saber reconocer y utilizar fuentes de información de diversos tipos y en una gran variedad de formatos, saber localizarlas y acceder eficientemente a la información. Sin embargo, no poseen un elevado nivel de destreza en cuanto a estas habilidades: solo el $41 \%$ de los encuestados declaró tener un dominio alto de todos los aspectos incluidos dentro de la búsqueda de información:

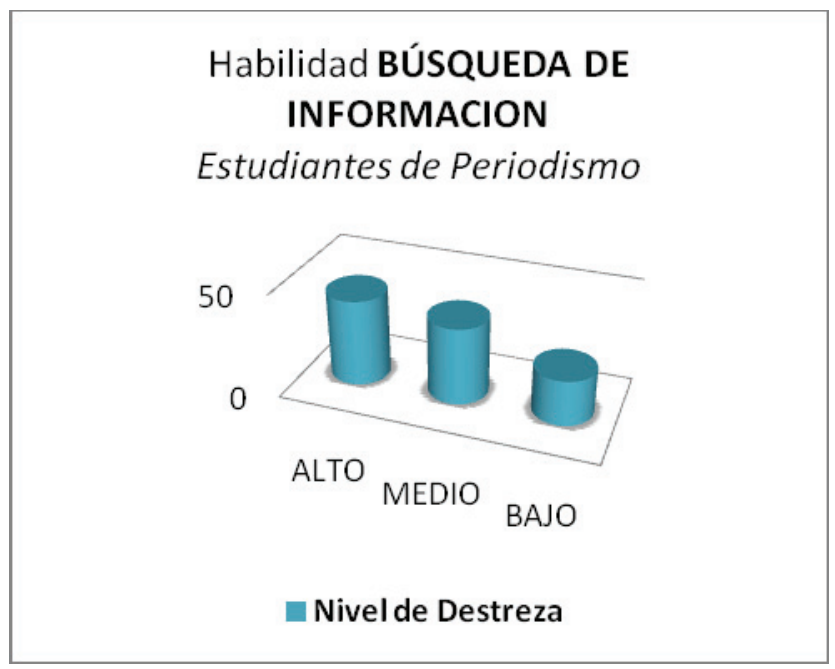

Fig. 1 Nivel de Destreza en la Búsqueda de Información (estudiantes periodismo)

Dentro de los indicadores de la habilidad búsqueda de información, resaltan cuestiones interesantes entre las respuestas (Fig. 27). Las interpretaciones a este fenómeno pueden ser diversas. Para los encuestados saber buscar y recuperar información en internet aparentemente no tienen relación con el dominio de estrategias de búsqueda de información o con la utilización de fuentes electrónicas como las bases de datos - ambos verificados con el coeficiente de Pearson 0.121 y 0.302 -, lo que 
resulta inexplicable pues cómo podrán hacer una búsqueda efectiva sino no combinan lo expresado en los ítems anteriores.

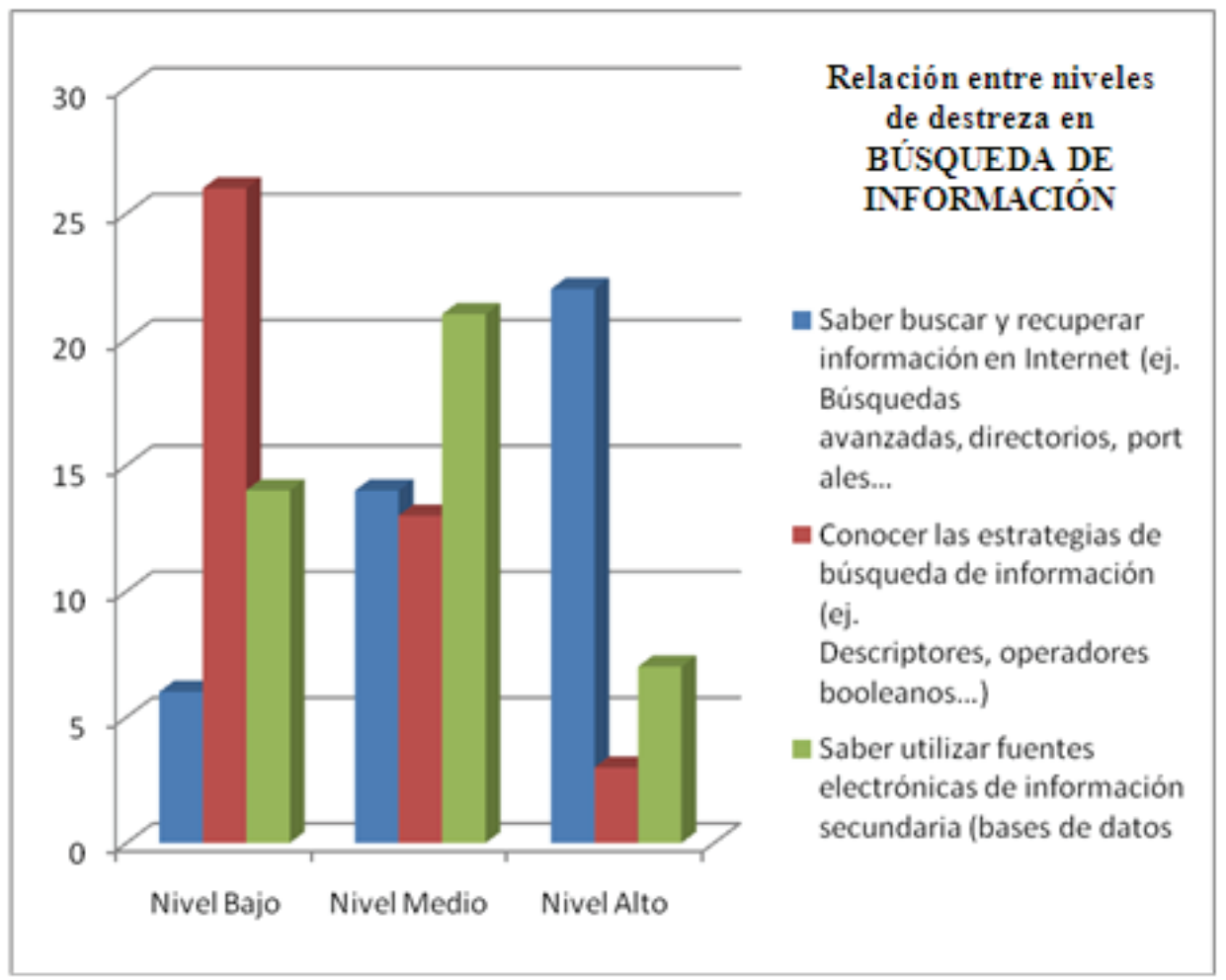

Fig. 1 Relación entre items de la búsqueda de información (estudiantes de periodismo)

La constatación del bajo nivel que poseen los estudiantes de periodismo en estos indicadores se expresa también a través de otros parámetros estadísticos. La media (5.95) de los estudiantes refiere sentirse regular en la búsqueda en Internet por medio de la utilización de búsquedas avanzadas o de los directorios, mientras que si se compara con el conocimiento de descriptores y estrategias de búsqueda la media decae hasta el rango de sentirse mal (3.12) pero si se analiza la desviación típica - 1.97 y 2.06- de ambos indicadores se puede constatar la gran dispersión en los resultados, lo que hace inferir que el dominio de la habilidad tiende a fluctuar entre medio y bajo. A pesar de la importancia que le atribuyen a estas habilidades, la generalidad de los estudiantes no se preocupa por adquirirlas mediante cursos o en las bibliotecas, pues el $49 \%$ de ellos declara haberlas adquirido de forma individual y el 34\% en el aula. En cuanto a la evaluación de la información, los futuros corresponsales consideran que tiene una gran importancia. Tan solo un $1 \%$ de los encuestados la calificó de innecesaria o poco importante. Esta es una de las competencias que mejor dominan: el $70 \%$ de los 
estudiantes afirma tener una alta destreza que han adquirido fundamentalmente en el aula y de forma individual.

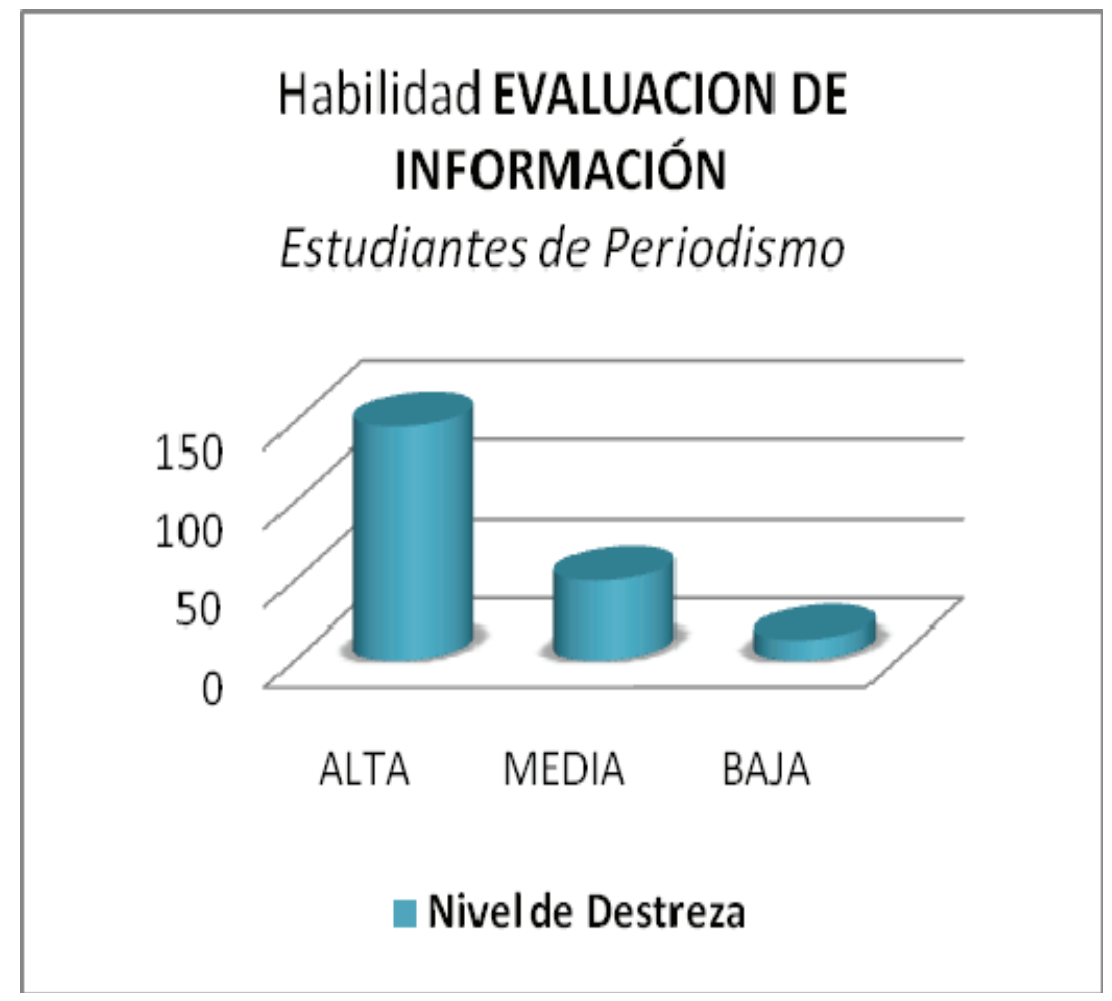

Fig. 3 Nivel de destreza en la Evaluación de Información (Estudiantes Periodismo)

Pese a esta idea, existe un desequilibrio en la relación entre los indicadores de la habilidad de Evaluación de la Información. Para los encuestados reconocer en el texto las ideas del autor tiene un peso fundamental para emitir juicios de valor con respecto a la información. Según los resultados que arrojó la tabla 3 del Anexo 12, donde con mayor fuerza ellos vislumbran esa dependencia es en este indicador; en un segundo plan favorecen que tener la capacidad de determinar si la información es actualizada también incide positivamente en evaluar con eficacia. No obstante, no interiorizan que el conocimiento de autores o instituciones tenga alguna relación, de hecho para ellos muy poca relación, dentro de la mencionada habilidad, solo fue significativo esta relación con la determinación de la actualidad de una fuente. Este elemento denota en ellos lagunas en el conocimiento de las habilidades de información necesarias de enfatizar sobre todo en el seno del aula pues es donde más la han adquirido. 


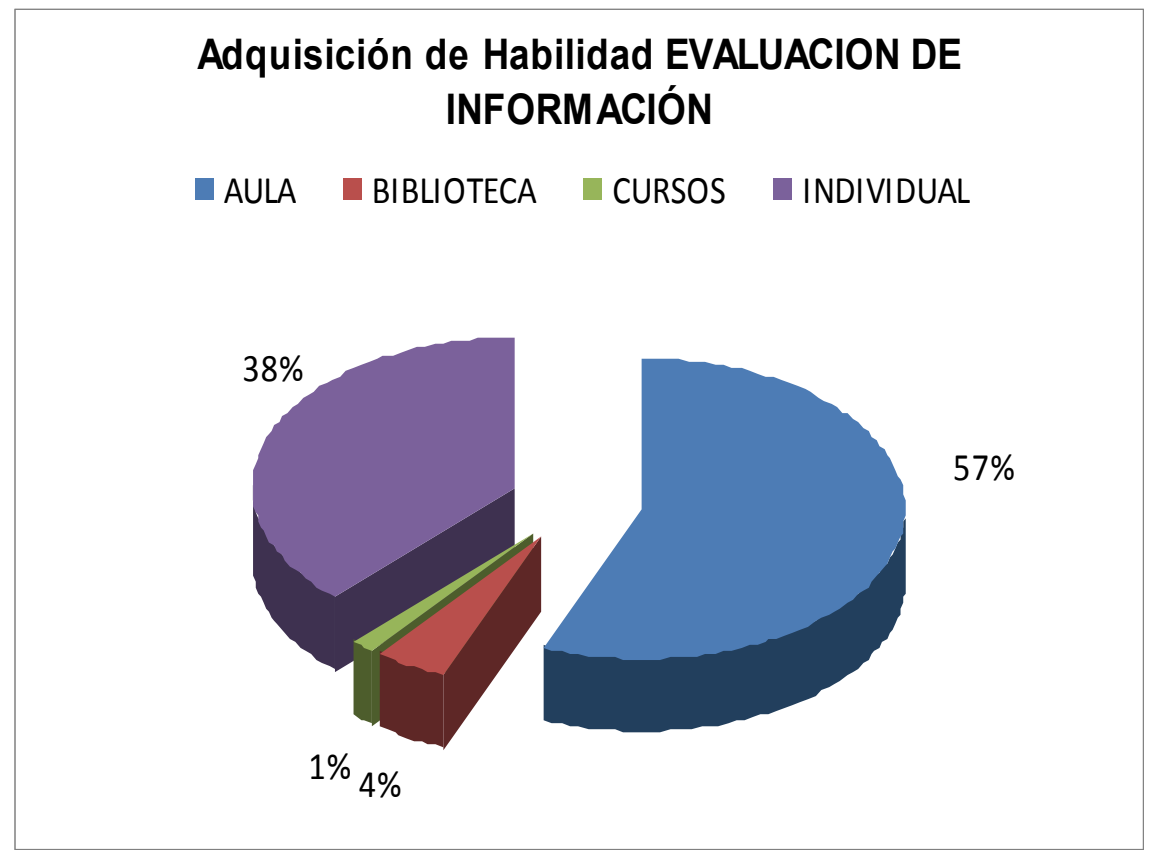

Fig. 4 Lugar de Adquisición de la habilidad Evaluación de Información (Estudiantes Periodismo)

Aunque en el grupo focal afirmaron sentirse especialmente débiles en todos los aspectos relacionados con la evaluación de la información, no se debe olvidar que fue realizado solo con alumnos de 4to año, grupo con una matrícula de 20 estudiantes e inferior a la de 5to año. La encuesta fue aplicada a los dos grupos, por tanto, sus resultados responden a ambos.Con respecto al tratamiento de la información, la mayoría de los encuestados lo considera muy importante. Contraproducente resulta, entonces, que no poseen un elevado nivel de destreza en esta habilidad. Sin embargo, no recurren a cursos ni a la biblioteca para revertir tal situación. El 47\% de los alumnos se conforma con adquirir las competencias necesarias para el tratamiento de la información en el aula y un $40 \%$ lo hace de forma individual. 


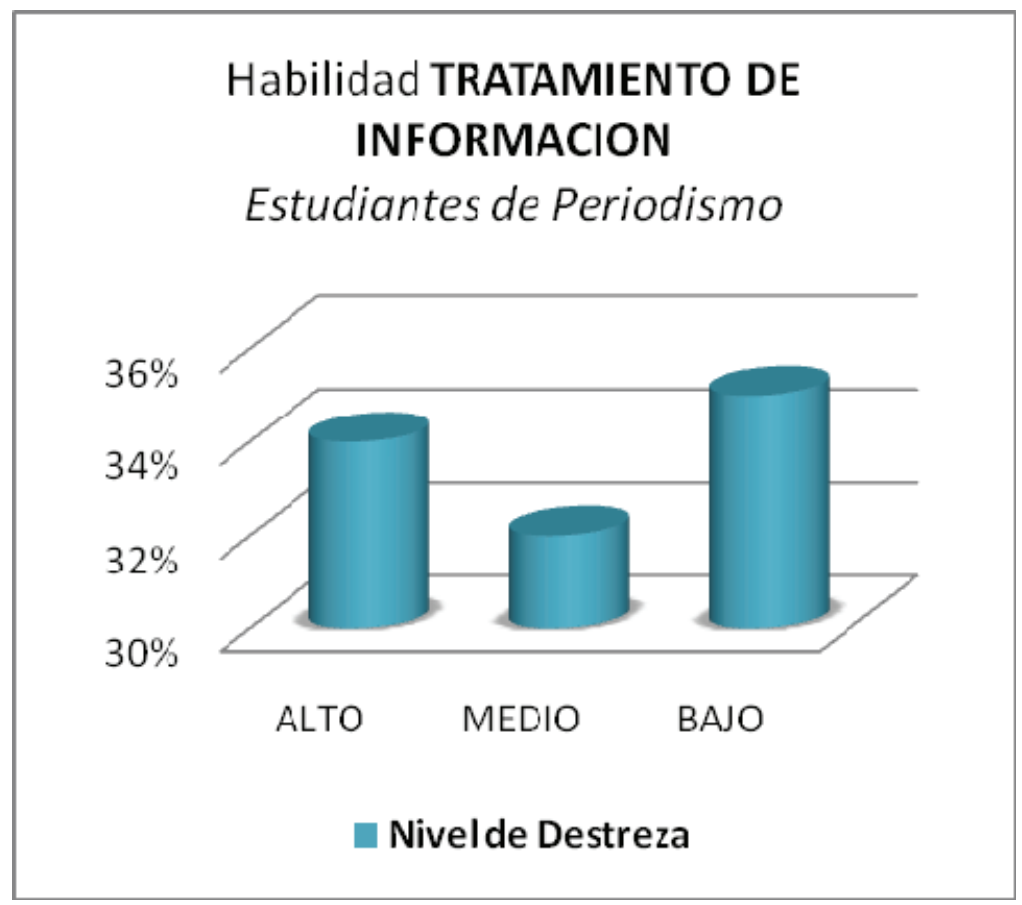

Fig. 5 Nivel de Destreza en el Tratamiento de la Información (Estudiantes Periodismo)

En esta habilidad aparecen indicadores muy depreciados en sus niveles de destreza, por ejemplo el $81 \%$ refiere sentirse entre un nivel medio y bajo con respecto a saber utilizar gestores de referencias bibliográficas (Ej. Endnote... ), incluso la moda calculada establece el valor 1, lo que indica que no saben en lo absoluto trabajar con software de este tipo. Esto fue un aspecto preocupante y que dentro de la asignatura Documentación Periodística se reforzó de forma directa. Así mismo afirman no saber manejar programas estadísticos y hojas de cálculo - la moda fue valor 1- y el $85.7 \%$ de los encuestados se catalogan entre medio y bajo nivel de destreza. Sin embargo le confieren una significativa relación -0.417 coeficiente de Pearson al nivel significante de 0.01- con respecto a la importancia que para el ejercicio de su profesión tiene dominar este acápite. Este elemento denota también la insuficiente efectividad de este rubro desde el aula pues el $47,6 \%$ de los estudiantes reafirma haberla adquirido desde el plano curricular.

A tono con la necesidad de utilizar las TICs en el ámbito periodístico para la efectividad del trabajo de los reporteros, se constató una gran debilidad en los niveles de destreza en el tratamiento de la información que implicaba uso de la TICs por aquellos indicadores no mediados por las TIC. Los estudiantes se sentían entre 81 y $88 \%$ en los niveles medio y bajo con respecto a varios ítems $-\mathrm{n} 16, \mathrm{n} 17, \mathrm{n} 18, \mathrm{n} 19-$, siendo en la mayoría de los casos inexistente la habilidad (Tabla 7 del anexo 12) 
La comunicación y difusión de la información fue otro de los aspectos medidos por la encuesta. En este punto incluimos cuestiones referidas tanto a la presentación de la información como a la ética y el respeto en el uso de la misma. Casi la totalidad de los estudiantes refirió concederle gran importancia a esta habilidad a la vez que aseguran poseer un alto nivel de destreza en cuanto a su dominio:

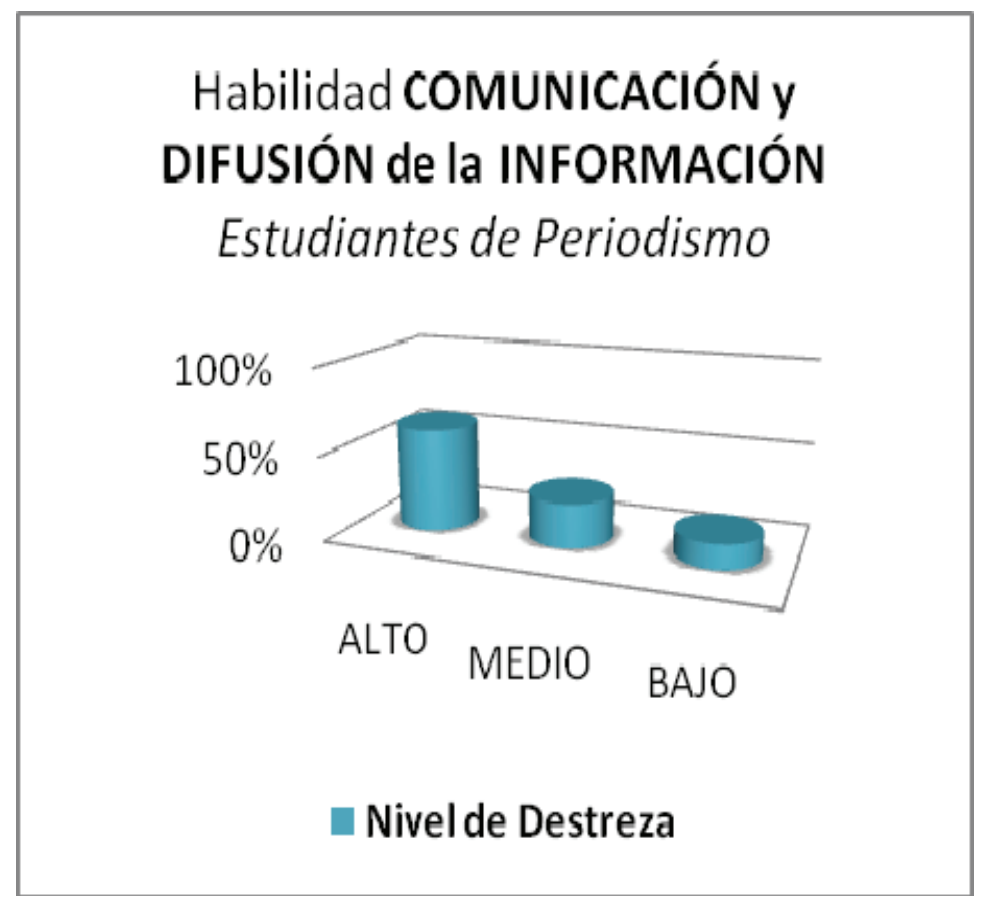

Fig. 6 Nivel de Destreza en Comunicación y Difusión de Información (Estudiantes Periodismo)

Han aprendido a comunicar y difundir la información mayormente de forma individual y en las aulas. Tan solo un $3 \%$ de ellos lo ha aprendido en la biblioteca y otro $5 \%$ en cursos.

Con respecto al conocimiento de la legislación sobre el uso de la información y de la propiedad intelectual, ítem específico en la encuesta y de vital importancia para el periodismo, los resultados fueron poco alentadores: el $64.3 \%$ de los estudiantes refirió poseer entre un nivel medio y muy bajo de destreza en esta habilidad.

Depreciado también se encuentra el nivel de destreza en la comunicación en otros idiomas, vital para cualquier reportero del siglo XXI. El 73.8\% posee un nivel entre medio y bajo, sin embargo la media se encuentra en 4.74 lo pudiera interpretarse como que los encuestados tienen un nivel medio, sin embargo al contraponerlo con la desviación típica de 2.64 se confirma la dispersión en los resultados y por ende su mala situación al existir estudiantes que están incomunicados fuera de su lengua materna. 
De manera general los futuros periodistas reconocen la importancia que todas las competencias en información tienen para el desarrollo de su profesión. No obstante, las adquieren fundamentalmente de forma individual o en el aula. La biblioteca y los cursos que muchas veces esa misma institución oferta quedan relegados a un segundo plano. En cuanto a la destreza en las habilidades de información, la evaluación es la que posee un nivel más alto, pues el $70 \%$ de los encuestados afirmó dominar todos los aspectos relacionados con la misma, respetando las interioridades o particularidades de cada habilidad.

Entre las necesidades que consideran más relevantes para obtener una mejor competencia informacional en su formación académica incluyeron, reiteradamente:

- "... que nos enseñen a utilizar las fuentes de información de manera real."

- "Mayor rigurosidad en la práctica académica para sistematizar los conocimientos."

- "Mayor acceso a Internet y mejores clases de las asignaturas relacionadas con la información."

- "Implementación de programas o cursos sobre la obtención y manejo de la información con las nuevas tecnologías de la información y las comunicaciones."

- "Mayor preparación por parte del claustro de profesores."

- "Ubicar de forma fija las clases de Documentación Periodística y profundizar en ellas."

- "La necesidad de que se profundice en la enseñanza del manejo de la información."

- "Mejor y más consciente acercamiento de la carrera a las nuevas tecnologías de la información y las comunicaciones."

\section{Resultados de la Fase 2: Sesiones desde las asignaturas}

En la asignatura se pudo actuar para incidir en cambios de postura y en el mejoramiento de las habilidades de información que estaban concebidas en los planes de estudios. Las transformaciones realizadas estuvieron en concordancia con los programas analíticos existentes (Anexos) y se tomaron en consideración las habilidades más depreciadas por los estudiantes en el diagnóstico. La orientación de actividades de trabajo independiente y en el aula fue concebida de manera que obligaran a los estudiantes a formar o consolidar las habilidades de información que encierra este proceso.

En el caso de Documentación Periodística el tema 2 estaba dirigido completamente a la formación y aplicación de las habilidades de información en el escenario periodístico. Las sesiones dedicadas a la habilidad de identificación de fuentes de información- estuvieron caracterizadas por el reconocimiento entre los estudiantes de las fuentes personales o directas, sin embargo no tenían identificadas otras fuentes y dentro del formato electrónico poseían un rango muy estrecho. Por ejemplo, se dedicó una clase a estudiar las clasificaciones de fuentes según el autor Andrés Cruz Paz ${ }^{4}$ a

\footnotetext{
${ }^{4}$ Cruz-Paz, Andrés (1996) Fuentes de Información. La Habana: Pablo de la Torriente, 92p.
} 
partir de aquí reflexionaron sobre la importancia de las bibliografías para el trabajo periodístico, fuentes como los repositorios institucionales lo escucharon por primera vez. No conocían la Web de la Ciencia y cómo existía un grupo de publicaciones sobre comunicación y periodismo. Otra de las sesiones se produjo en el laboratorio de la biblioteca donde tuvieron que buscar en el sitio web del CDICT aquellas fuentes que podían servirle para su especialidad. En internet fueron conducidos hasta el sitio www.internetinvisible.com y pudieron familiarizarse con diversas fuentes de información.

En la primera sesión dedicada a la localización y búsqueda de información y ante una interrogante de la investigadora, según sus propios comentarios "estamos en pañales, profe". Sus manifestaciones en el aula eran que para encontrar algo, "ponemos toda una oración en el google". Por tanto, se inició con una descripción desde el punto de vista teórico sobre el proceso de búsqueda, tipos de búsqueda etc; admitieron escuchar por vez primera qué era una estrategia de búsqueda y un perfil de búsqueda. El resto de las sesiones de este tema concurrieron en el laboratorio, donde ejercitaron la aplicación de los operadores booleanos en la base de datos EBSCO y el sitio www.scirus.com disponibles desde la biblioteca. También accedieron al catálogo automatizado de la biblioteca y aplicaron los operadores, donde ellos mismos detectaron las imperfecciones del sistema. La última de la sesiones fue evaluativa, donde tenían que exponer de un tema escogido por ellos qué fuentes podían servirle y cómo llegaron a localizarlas. Hubo un estudiante que llegó hasta el repositorio de la Universidad Carlos III de Madrid, al departamento de comunicación y encontró trabajos investigativos sobre la fotografía, de gran importancia para su futuro trabajo de diploma.

En el grupo focal ellos admitieron "para nuestro trabajo el peso principal lo tienen las fuentes no documentales, especificamente las personas y la forma de validar que nos dan es tener en cuenta si es una fuente oficial, si es autorizada o si es testigo del hecho". Más adelante manifestaron" nada, no nos han enseñado ningún criterio de evaluación, mucho menos con respecto a Internet". Por ende, las sesiones de evaluación de la información fueron muy dinámicas a través de talleres. Se concentraron en valorar formalmente las fuentes- autoría, prestigio de las editoriales, cantidad de reimpresiones, más que emitir juicios sobre el contenido, basados en criterios como la veracidad y relevancia de la información y sobre todo se enfatizó en los elementos a considerar en una fuente electrónica, por ejemplo diferenciar la última fecha de actualización de la página con respecto a la fecha del copyright. Fueron sesiones de varias anécdotas y experiencias de muchos con respecto a este tema.

En las sesiones de organización de la información, más que hacer fichas de contenidos, que ellos afirmaron realizar con frecuencia y por tanto se sentían con mayor preparación, se concentraron en el adiestramiento con el Endnote como gestor bibliográfico existente en la UCLV. Se dedicaron varias clases práctica a familiarizarse con el mencionado gestor. Aquí, fueron más lentos en aprender a trabajar y comprender la relación entre el Endnote y el microsoft word. Además se detuvo la investigadora en explicar los estilos bibliográficos, enfatizando en el uso del estilo Harvard, pues es el exigido en la carrera para los trabajos de curso y diploma, cómo citar dentro del texto y 
cómo hacer las listas de referencias. Se revisaron en el aula diversos ejemplos traídos por los propios estudiantes y que fueron aclarados por otros ej. Cómo citar una entrevista personal. La evaluación de este tema consistía en la entrega de una biblioteca Endnote y presentarla ante la profesora /investigadora y realizar operaciones con la biblioteca en tiempo real.

Las últimas sesiones de la asignatura fueron para retomar y repasar los elementos de comunicación oral y escrita, así como para debatir los elementos éticos legales asociados al uso de la información.

\section{Resultados de la fase 3: Las acciones con los estudiantes}

A partir de las acciones realizadas se lograron constatar cambios, que si bien no tan radicales, pueden valorarse como avances en sentido general en los encuestados. En el caso de Periodismo fueron muestras del avance las reflexiones realizadas en los ensayos finales de la asignatura Documentación Periodística que apuntan la profundidad en el tratamiento de las diferentes habilidades de información, argumentos como:

- La credibilidad de un medio está en relación directa con la cantidad, calidad y diversidad de las informaciones y de las fuentes que en él se citan. Cuantas más fuentes se consulten, más diversa será la información que se publica. Disponer de un número ilimitado de fuentes significa para el periodista un incremento del prestigio dentro del gremio [...] (estudiante periodismo)

- El mensaje periodístico que se produce en los medios internacionales actuales muchas veces se ve hipotecado por mañas obcecadas, pues los profesionales de la información no identifican debidamente a sus fuentes y son propensos a propagar una versión deformada de la realidad (estudiante periodismo)

- Esta modalidad del periodismo -refiriéndose al periodismo de investigaciónadmite desde el trabajo con fuentes institucionales, privadas, hasta la búsqueda de documentos impresos, de grabaciones de audio o video, bases de datos o sea fuentes de información digitales, en fin toda una amplia gama que permita hallar la verdad del asunto en cuestión. [...] (estudiante periodismo)

- Un consumo antiético de la información, como hemos visto, y su posterior difusión, conduce inevitablemente a una marcada deformación de sucesos, que si es descubierta produce en el público un alto grado de desconfianza y desconcierto. Cuando se descubre falsedad o engaño deliberado en el empleo de la información, el perjuicio se vuelve contra la institución y el periodista, cayendo ambos en una zona de descrédito. [...] (estudiante periodismo)

- Hoy, ¿quién toma por veraz un hecho donde no haya un notable cotejo de fuentes? ¿Quién no valora la actualidad usando como referencia las fuentes tratadas por el periodista? ¿Cómo se establece la proximidad en un trabajo de prensa, sino mediante el uso de fuentes con modelos de pensamientos más cercanos a los receptores? [...] (estudiante periodismo)

- Aunque la "calidad" de la información sí se evalúa, pero de forma general; más interesa la calidad si es "gracioso", "entretenido" o "bello" el sitio visitado, en lugar de analizar cuán instructiva o válida es la información que con- 
tiene.[fragmento para referirse a los criterios para evaluar información empleado por los estudiantes universitarios]

La exhaustiva explicación en un examen de premio $^{5}$ de la propia asignatura sobre el proceso de búsqueda de información desarrollado por una estudiante, también confirma la idea de transformación y aprehensión de conocimientos sobre el tema con la aplicación de las habilidades de información abordadas en la asignatura:

Tema de tesis en frase concreta:

Tratamiento periodístico de la cuestión nacional...

\section{Estrategias de búsqueda:}

La primera categoría de la investigación constituye un concepto suficientemente tratado, por tanto, puedo proceder a través de operadores absolutos como las comillas ("tratamiento periodístico") u operadores lógicos como (tratamiento AND periodístico), pues los resultados resultan similares; con otros operadores la búsqueda se aleja de su objetivo.

La segunda categoría de la investigación constituye un concepto sistematizado por mi tutor, pero para asumirlo, debo analizar las formulaciones anteriores, aunque cada una de ellas ofrece porciones de la cuestión nacional con que trabajo. O sea, aquí las propuestas de búsqueda resultan similares: (por operadores absolutos: “cuestión nacional” u operadores lógicos: cuestión AND nacional. Con estos operadores o con uno de truncamiento como (cuestión + nacional) puedo conseguir informaciones, pero con mayor similitud al concepto de cuestión nacional asumido en la investigación aparecen términos como nacionalismo, nación y soberanía que pudieran enriquecer la búsqueda y a los cuales les puedo aplicar iguales operadores de búsqueda.

Aclaración: El uso del perfil de búsqueda parece ser más útil para cuando la investigación esté más adelantada y se necesite información más detallada sobre algún asunto especifico, pues presupone no sólo del objeto de búsqueda, sino también del autor.

\section{Fuentes de información para localizar materiales relativos al tema:}

De acuerdo con el criterio de portador de la información: fuentes documentales primarias como tesis, secundarias como fichas del catálogo y terciarias como bibliografias.

De acuerdo con el criterio de la forma de representación y registro fisico de la información en el soporte informacional: textual y electrónica.

De acuerdo con el criterio de la forma convencional de reproducción: en su mayorias, publicadas, pero también si llega a ser pertinente, las no publicadas, como tesis o libros, también resultaría útiles.

${ }^{5}$ El examen de premio constituye una vía para elevar la calidad de los egresados que forma la Educación Superior, y tienen como objetivo estimular que los estudiantes profundicen en el estudio de las asignaturas y disciplinas que conforman su plan de estudio.Artículo 195. MES. RESOLUCIÓN 210/07. Reglamento para el Trabajo Docente y Metodológico en la educación superior. Ciudad de La Habana: 2007. 
Otra de las acciones del proceso de evaluación fue la presentación en el colectivo de la carrera de Periodismo de los resultados del estudio realizado y propiciar el análisis de la asignatura y si incursión en años precedentes. Este aspecto fue valorado en el diseño del plan D de la especialidad donde en el currículo propio de la Universidad Central y se decidió ubicar la asignatura en el 2 do año de la especialidad, con el propósito de contar con más tiempo dentro de la vida universitaria para trabajar desde todas las asignaturas posibles el desarrollo de las habilidades de información.

\section{CONCLUSIONES}

1. El periodismo como especialidad trabaja directamente con la información, lo cual impone su uso eficiente por los futuros profesionales. La preparación desde las aulas universitarias, se convierte hoy en una prioridad.

2. La autovaloración que tienen los estudiantes incluidos en la investigación arrojó tener niveles medio y bajos de destreza de habilidades de Información. Particularmente el 66\% y 59\% afirman encontrarse en estas categorías en el Tratamiento y la búsqueda de la Información respectivamente.

3. El escenario docente de la asignatura documentación periodística fue determinante en la transformación de la realidad encontrada en el diagnóstico. Las opiniones dadas por los estudiantes en cada sesión, así como las evaluaciones finales demostraron el avance de los encuestados con respecto al desarrollo de las habilidades de la información.

\section{REFERENCIAS BIBLIOGRÁFICAS}

BENITO, F. Nuevas necesidades, nuevos problemas. Fundamentos de la alfabetización en información. En: Gómez, J. A. (2000) Estrategias y modelos para enseñar a usar la información: guía para docentes, bibliotecarios y archiveros. Murcia, Editorial KR, pp. 11-75.

BORSTEIN, J. Journalism students and information competencies. Academic Exchange, 2003.

BRADLEY, F. Information Literacy and news libraries: the challenge of developing information literacy instruction programs in a special library environment. Media and Information. Curtin University of Technology, 2003.

BURKHARDT, J. M. Assessing Library Skills:A First Step to Information Literacy. Portal: Libraries and the Academy, 2007, vol. 7, $\mathrm{n}^{\circ}$ 1, p. 25-49

CARRISON, B. Journalist's percepcion of online information-gathering problems. Journalism ans Mass Comunication Quaterly, 2000, vol. 77, $\mathrm{n}^{\mathrm{o}} 3$, p. 500-514

COLBORN, N. W. y CORDELL, R. M. Moving from Subjective to Objective Assessments of Your Instruction Program. Reference Services Review, 1998, vol. 26, no 3-4, p. 125-137 
ERICSON, R. V.; CHAN, J. B. L., et al. Negotiating control: a study of news source. Milton Keynes: Open University Press, 1989.

GRATCH LINDAUER, B. The Three Arenas of Information Literacy Assessment. Reference \& User Services Quarterly, 2004, vol. 44, no 2, p. 122-129

GUALLAR, J. La renovación de los diarios digitales: rediseños y web $2.0 \mathrm{El}$ profesional de la información, 2007, vol. 16, $\mathrm{n}^{\circ}$ 3, p. 235-242

HERNÁNDEZ SAMPIER, R.; FERNÁNDEZ-COLLADO, C., et al. Metodología de la investigación. Editado por: Edición, T. México, D.F: McGraw- Hill, 2006.

IANNUZZI, P. We are teaching, but are they learning: accountability, productivity, and assessment. The The Journal of Academic Librarianship, 1999, vol. 25, $\mathrm{n}^{\mathrm{o}}$ 4, p. 304305

IFLA. Directrices para la evaluación de la alfabetización informativa [Consultado el: 10 enero de 2006]. Disponible en: http://www.ifla.org/VII/s42/pub/ILguidelines2004-s.pdf.

KUNKEL, L. R.; WEAVER, S. M., et al. What Do They Know?: An Assessment of Undergraduate Library Skills. The The Journal of Academic Librarianship, 1996, vol. $22, n^{\circ} 6$, p. $430-434$

LARKIN, J. E. y PINES, H. A. Developing information literacy and research skills in introductory Psychology: a case study. The The Journal of Academic Librarianship, 2005 , vol. $35, \mathrm{n}^{\circ} 1$, p. $40-45$

LAWSON, M. D. Assessment of a college freshman course in information resources. Library Review, 1999, vol. 48, n ${ }^{\circ}$ 2, p. 73-78

MES. RESOLUCIÓN 210/07. Reglamento para el Trabajo Docente y Metodológico en la educación superior. Ciudad de La Habana: 2007.

MOORE-JANSEN, C. What Difference Does It Make? One Study of Student Background and the Evaluation of Library Instruction. Research Strategies, 1997, vol. $15, \mathrm{n}^{\mathrm{o}} 1$, p. $26-38$

MORRISON, H. Information Literacy Skills:An Exploratory Focus Group Study of Student Perceptions. Research Strategies, 1997, vol. 15, no 1, p. 4-17

O' REILLY, T. What is web 2.0: design patterns and business models for the next generation of software. [Consultado el: 23 marzo de 2009]. Disponible en: http://www.oreillynet.com/go/web2.

PINTO MOLINA, M. Design of the IL-HUMASS survey on information literacy in higher education: A self-assessment approach. Journal of Information Science, 2010, vol. $36, \mathrm{n}^{\circ} 1$, p. $86-106$

PORTMANN, C. A. y JILIUS ROUSH, A. Assessing the effect of library instruction. The Journal of Academic Librarianship, 2004, vol. 30, $n^{\circ}$ 6, p. 461-465

RABINE, J. y CARDWELL, C. Start making sense: Practical approaches to outcomes assessment for libraries. Research Strategies, 2000, vol. 17, $\mathrm{n}^{\mathrm{o}}-$, p. 319-335

RODRÍGUEZ GOMEZ, G.; GIL FLORES, J., et al. Metodología de la investigación cualitativa. La Habana: Félix Varela, 2004.

SINGH, A. B. A Report on Faculty Perceptions of Students' Information Literacy Competencies in Journalism and Mass Communication Programs: The ACEJMC Survey. College \& Research Libraries, 2005, vol. 66, nº 4, p. 294-311 
UNESCO.Model curricula for journalism education for developing countries \& emerging democracies.2007.Series on Journalism education

WONG, G.; CHAN, D., et al. Assessing the Enduring Impact of Library Instruction Programs. The Journal of Academic Librarianship, 2006, vol. 32, nº 4, p. 384-395 